\title{
Research on the Composite Braking Control of Electric Vehicle
}

\author{
Jinglei Dou, Gaojian Cui, Shaosong Li*, Shunhang Zheng, Xiaoquan Zhu and Zhixin Yu \\ School of Mechatronics Engineering, Changchun University of Technology, Changchun 130012, China \\ ${ }^{*}$ Corresponding author
}

\begin{abstract}
A new composite braking control strategy is proposed for a rear-wheel-drive electric vehicle $(\mathrm{EV})$ with both a regenerative braking system and a hydraulic braking system to achieve improved braking performance and optimal energy regeneration. Based on the model predictive control (MPC), the proposed braking control strategy maintains the slip ratio within an optimal range by adjusting the braking torque continuously, besides, to recover optimal braking energy, a braking torque allocation strategy of maximum regeneration efficiency is designed. With the control strategy, the regenerative braking system and the hydraulic braking system work synchronously to assure high regenerative efficiency and good braking performance. The proposed braking control strategy is steady and effective, as demonstrated by the simulation.
\end{abstract}

Keywords-composite braking; slip ratio; electric vehicle (EV); model predictive control (MPC); regenerative braking.

\section{INTRODUCTION}

Energy regeneration during braking is important for electric vehicle (EV) because it allows the vehicle to improve its fuel economy and extend its driving range. During braking, with the regenerative system of $\mathrm{EV}$, the kinetic energy is converted to electric energy, so that it can be stored in an energy storage unit such as a battery for reuse when driving. However, when regenerative braking is applied to actual vehicles, the required braking force may not be guaranteed because of many constraints[1]. therefore, the composite braking of regenerative braking and hydraulic braking is crucial for brake comfort and brake safety as well as for regeneration efficiency.

As for the composite braking, few investigations have been reported, and how to adjust the relationship between regenerative braking system and hydraulic braking system is still an important issue to research on EV. Present research mainly concentrates on two different braking scenarios[2]. One is the normal deceleration process. Research in [3] used the sliding-mode control method, modulating the hydraulic braking force to obtain the maximum regeneration efficiency and good brake comfort. A hierarchical control strategy for cooperative braking system of an electric vehicle was put forward in [4], in which, the top layer is used to optimize the braking stability based on two sliding mode control strategies, and the bottom layer is used to maximize the regenerative braking energy recovery efficiency with a reallocated braking torque strategy. The other focus is the emergency braking process. To deal with the rapid deceleration and the altered friction braking torque, a concurrence control and a compensation control were proposed in [5]. A new braking torque distribution strategy was proposed based on the required braking torque and the regenerative braking system's status in [6].

However, these studies have limitations in dealing with constrained problem and can not realize the optimal braking torque for each wheel. To achieve the functions above, this paper developed a model predictive controller to maintain the slip ratio within an optimal range by calculating the optimal braking torque continuously, and then designed a braking torque allocation strategy to recover the maximum regeneration energy. This proposed control strategy can guarantee both the optimal braking torque on each wheel and the maximum regeneration efficiency. The braking effectiveness and regeneration efficiency of the proposed control strategy demonstrated through the simulation.

\section{SYSTEM MODEL}

\section{A. Vehicle Dynamic Model}

Consider a rear-wheel-drive vehicle motion when braking operation is performed on a flat road. Ignoring the air resistance and rolling resistance, assuming no steering, only the longitudinal motion is considered. The dynamic differential equations can be expressed as follows.

$$
\begin{gathered}
m \dot{v}=F_{x f l}+F_{x f r}+F_{x r l}+F_{x r r} \\
J \dot{\omega}_{i j}=F_{x i j} r-T_{b i j} \quad(i=f, j=l, r) \\
\left(J+\frac{1}{2} J_{p t}\right) \dot{\omega}_{i j}=F_{x i j} r+T_{d i j}-T_{b i j} \quad(i=r, j=l, r)
\end{gathered}
$$

where $m$ is the vehicle mass; $v$ is the longitudinal velocity; $J$ is the moment of inertia of tire; $J_{p t}$ is the moment of inertia of the powertrain which is shared by the two drive wheels; $\omega$ is the wheel angular speed; $r$ is the wheel radius; $F_{x i j}$ is the friction force; and $T_{d i j}, T_{b i j}$ are the driving and braking torques. In addition, $i j=f l$ for the front left wheel, $i j=f r$ for the front right wheel, $i j=r l$ for the rear left wheel and $i j=r r$ for the rear right wheel.

\section{B. Tire Model}

The tire model used in this paper is the "Magic Formula" model developed by Pacejka. In this model, the tire longitudinal force is described as the complex nonlinear function of the longitudinal slip ratio and tire vertical load. 


\section{Longitudinal Slip Calculation Model}

In this section, the longitudinal slip ratio can be written as Equation (4).

$$
\lambda=\frac{v-\omega r}{\max (v, \varepsilon)}
$$

where $\varepsilon$ is a small constant in order to avoid zero denominator. In this paper, the value of $\varepsilon$ is set as 0.1 .

By taking the differential of Equation (4), the slip dynamics can be expressed as Equation (5).

$$
\dot{\lambda}=\frac{\dot{v}}{v}(1-\lambda)-\frac{\dot{\omega} r}{v}
$$

\section{Motor Model}

The power into the motor can be calculated as Equation (6).

$$
P_{r}=T_{M} \cdot \omega_{r} \cdot \rho\left(T_{M}, \omega_{r}\right)
$$

where $P_{r}$ is the power generated by regenerative braking system; $T_{M}$ is the whole regenerative braking torque; $\omega_{r}$ is the wheel angular speed of rear and $\rho\left(T_{M}, \omega_{r}\right)$ denotes the power efficiency.

The transfer function of the electric motor of the first order can be written as

$$
G(s)=\frac{1}{0.03 s+1}
$$

\section{E. Battery Model}

The battery model is built as an open-circuit voltage model. The state of charge (SOC) and motor current are modeled as follows

$$
\begin{gathered}
S O C=S O C_{i n i}-\frac{\int_{0}^{t} I_{b}(\tau) d \tau}{Q_{c}} \\
I_{b}=\frac{U_{0}-\sqrt{P_{r} R_{b}}}{R_{b}}
\end{gathered}
$$

where $S O C$ is the actual battery SOC value; $S O C_{i n i}$ is the initial battery SOC value; $Q_{c}$ is the total battery energy capacity. $U_{0}$ is the battery open-circuit voltage; $R_{b}$ is the equivalent battery internal resistant; $I_{b}$ is the motor current.

\section{F. Hydraulic Braking Model}

The hydraulic system is described as pressure increasing, pressure holding, and pressure dumping processes. In this paper, we adopt the hydraulic system in [7]

\section{MPC-BASED COMPOSITE BRAKING CONTROLlER DESIGN}

In this section, a composite braking control strategy based on MPC is proposed. The primary task of this controller is to maintain the slip ratios within an optimal range by adjusting the optimal braking torques of four wheels continuously. The MPC-based control block diagram is shown as Fig. 1.

where $\lambda_{d}$ is the desired value of MPC slip controller module, $T_{H r l}, T_{H r r}, T_{M r l}, T_{M r r}$ are the hydraulic and regenerative braking torques of the two rear wheels respectively, and $\lambda_{f l}, \lambda_{f r}, \lambda_{r l}, \lambda_{r r}$ are the four tires' slip ratios.

As illustrated, the MPC slip controller module can predict the future condition of this system, and then output the optimized torque command to the EV model, to ensure that the real longitudinal slip can track the optimal one. The allocation strategy module after the MPC slip module to allocate the regenerative braking torque and hydraulic braking torque of composite braking system.

\section{A. Reference Input}

From the "Magic Formula", when $\dot{\lambda}=0$, the friction coefficient achieve the maximum, and the slip ratio is defined as $\lambda_{d}$ which denotes the slip ratio equilibrium point. That is to say, if slip ratio can be kept at $\lambda_{d}$ all times ideally except for the timing of slip increasing from 0 to $\lambda_{d}$ until the vehicle stops during braking, the optimal brake performance can be achieved. In this regard, we choose the slip ratio equilibrium point $\lambda_{d}$ as the desired value .i.e. the reference input. 


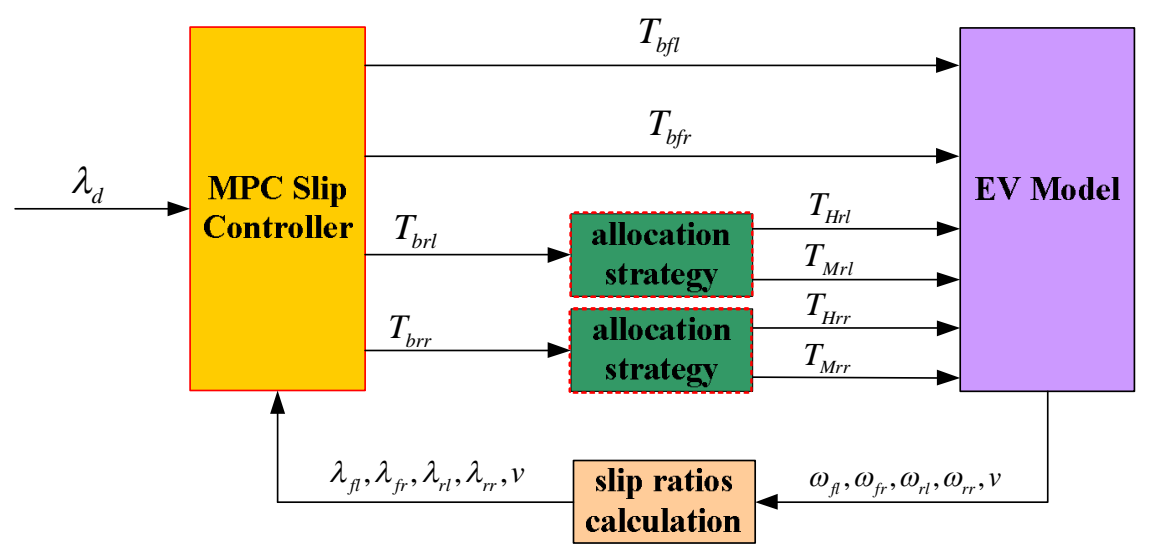

FIGURE I. BLOCK DIAGRAM OF THE MPC-BASED COMPOSITE BRAKING CONTROL SYSTEM.

\section{B. MPC Slip Controller Design}

Based on the slip control model (Equation (1)-(5)) of EV, a state-space model for MPC can be obtained, then a finite dimensional optimal control problem can be defined [8][9]. Let the state-space model derived as Equation (10)

$$
\begin{aligned}
& x(k+1)=A x(k)+B u(k) \\
& y(k)=C \cdot x(k)
\end{aligned}
$$

where $x=\left[\lambda_{f l}, \lambda_{f r}, \lambda_{r l}, \lambda_{r r}\right]^{T}$ is defined as the state variable, $u=\left[T_{b f l}, T_{b f r}, T_{b r l}, T_{b r r}\right]^{T}$ is defined as the control input, and $y=\left[\lambda_{f l}, \lambda_{f r}, \lambda_{r l}, \lambda_{r r}\right]^{T}$ is the system output.

The optimization object function of the optimal control problem at step $k$ can then be written as Equation (11).

$$
\begin{aligned}
\min _{u} J= & \sum_{i=1}^{P}\left\|\Gamma_{y, i}(y(k+i \mid k)-r(k+i \mid k))\right\| \\
& +\sum_{i=1}^{M}\left\|\Gamma_{u, i} \Delta u(k+i-1 \mid k)\right\|
\end{aligned}
$$

where $r(k+i \mid k)$ denotes the reference slip ratio $\lambda_{d}$ of four wheels; $y(k+i \mid k)$ denotes the slip ratio of four wheels, $\Delta u(k+i-1 \mid k)$ is the increment of slip ratio; $\Gamma_{y i}, \Gamma_{u i}$ are the weighting matrices; $P$ and $M$ are the predictive horizon and control horizon respectively, $P \geq M \geq 1$.

$$
J_{1}=\sum_{i=1}^{P}\left\|\Gamma_{y, i}(y(k+i \mid k)-r(k+i \mid k))\right\| \text { is to keep the real }
$$

slip ratio of each wheel within the optimal one to prevent the tires from locking up.

$$
J_{2}=\sum_{i=1}^{M}\left\|\Gamma_{u, i} \Delta u(k+i-1 \mid k)\right\| \text { is added to control the }
$$

changing rate of the control action to preserve the comfort of EV driver.

Take the maximum torque limitations and the value range of wheel slip into consideration, the constrains can be expressed as Equation (12).

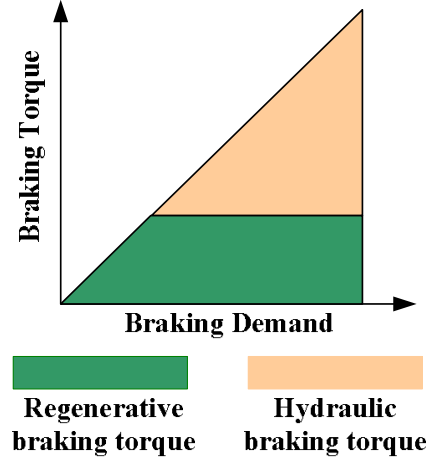

FIGURE II. DIAGRAM OF ALLOCATION STRATEGY

$$
\begin{aligned}
& \left|T_{i}(k+j \mid k)\right| \leq T_{\max }, j=0,1, \ldots, M-1 \\
& 0 \leq \lambda_{i}(k+j \mid k) \leq 1, j=0,1, \ldots, P .
\end{aligned}
$$

MPC solves an optimization problem online at each time instant, with knowledge of the current system state variables and previous system inputs, and then takes the first element of the optimal control sequence as the current control to the system.

\section{Braking Torque Allocation}

For rear-wheel-drive EV, the braking torques of four wheels have been calculated by MPC strategy. The braking torques of front wheels are supplied by hydraulic system alone, and the current task is to allocate the rear wheels' regenerative braking torque and hydraulic braking torque.

In this regard, a new braking torque allocation strategy is designed for rear wheel in view of regenerative efficiency. The diagram of allocation strategy is shown as Fig. 2.

As illustrated, this allocation strategy targets the maximum regenerative efficiency, the regenerative braking torque is utilized to its maximum extent, and the hydraulic braking torque will not be exerted on the rear wheel until the motor torque cannot meet the deceleration request. Theoretically, this strategy can maximize the use of the 
regenerative braking torque, achieve the maximum regeneration efficiency.

\section{SimUlation AND ANALYSIS}

Simulation is conducted in the MATLAB/Simulink environment to show the control performance of the proposed controller. According to the engineering structure of EV, arear-wheel-drive EV model is built in MATLAB/Simulink. The model mainly includes the motor model, the braking model, the battery model, the diver model, the tire model, and the roadwheel model, etc.

In this section, the simulation step is defined as $0.001 \mathrm{~s}$, the predictive horizon $P=10$ and the control horizon $M=5$. The road adhesion coefficient is set to 0.85 , and the initial speed is defined as $78 \mathrm{~km} / \mathrm{h}$.

From the simulation results of proposed controller shown in Fig. 3, the front braking torque has a fast increase at the first $0.25 \mathrm{~s}$. As for the rear wheel, at the beginning of the braking procedure, the regenerative braking torque increases smoothly, while the hydraulic braking has a short oscillation. After the regenerative braking torque increases to its maximum extent at about $0.5 \mathrm{~s}$, which can meet most of the rear braking demand, and the rear hydraulic braking torque is kept at a low value. As can be seen from Fig. 3(b), at the beginning of the braking procedure, the slip ratios increase rapidly and have a mall fluctuation because of the the sharply increasing braking torque. During this time, due to the big load of front wheel and the fast response of regenerative system of rear wheel, the front slip ratio oscillates severely. The slip ratios trace the reference slip ratio well at the rest time, and decrease to zero finally. Owning to the maximum supply of regenerative braking system, the SOC value increases quickly and almost reached the value 0.52 .

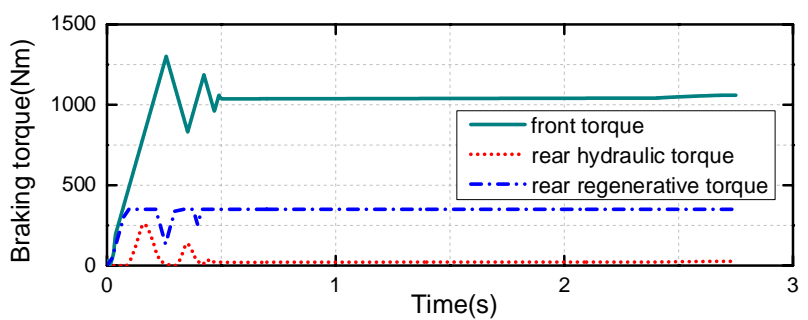

(a) Braking torques

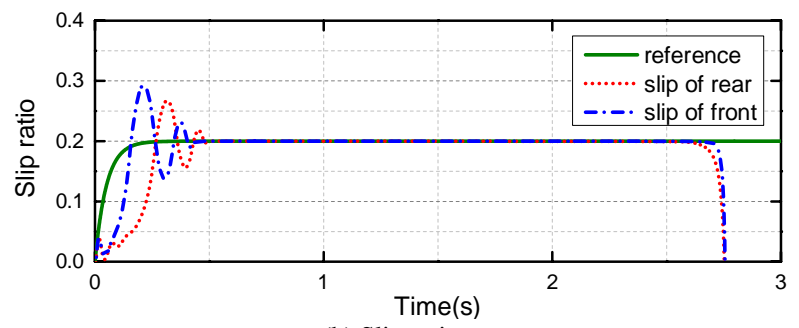

(b) Slip ratios

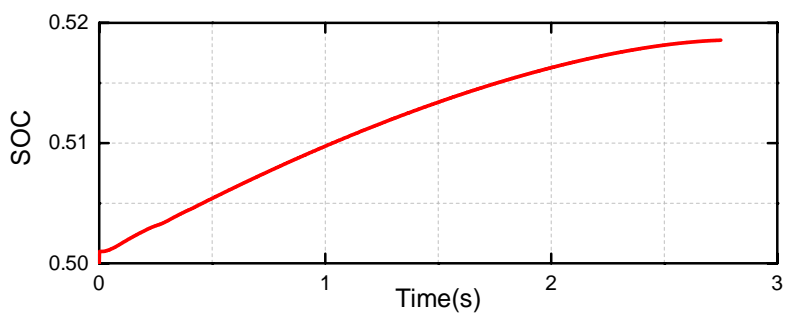

(c) $\mathrm{SOC}$

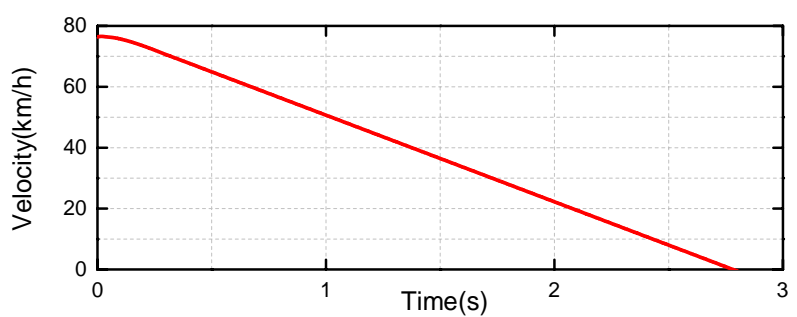

(d) Velocity

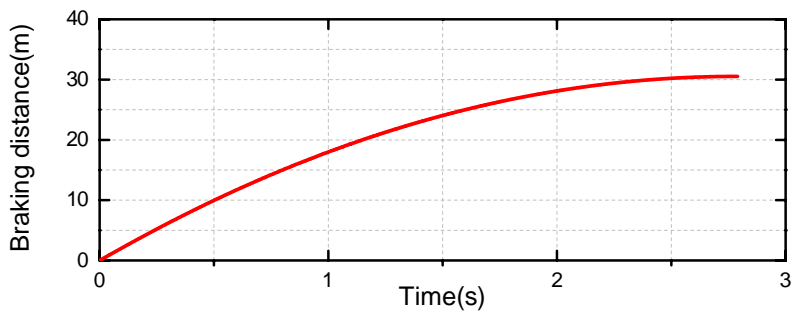

(e) braking distance

FIGURE III. SIMULATION RESULTS OF PROPOSED CONTROLLER

Speaking of the velocity, at the beginning of the braking procedure, velocity decreases slowly, and then it declines markedly. The braking distance ascends gently and finally reaches $30.5 \mathrm{~m}$.

\section{CONCLUSION}

In this paper a new composite braking control strategy is proposed to achieve the optimal braking torques and high regeneration efficiency. This proposed controller takes advantage of MPC to maintain the slip ratio within an optimal range by adjusting the braking torque continuously, and a barking torque allocation strategy is designed in view of the regeneration efficiency. The effectiveness of the proposed control system has been verified through simulation using an EV model which was built in Simulink. The results prove that this proposed controller can stop the vehicle from locking up and implement good braking efficiency, meanwhile, the maximum regeneration efficiency can be achieved.

\section{ACKNOWLEDGMENT}

The work is supported by the Jilin Province Science and Technology Found for Young Scholars (20170520097JH; No.20160520106JH), by Key Technology on Major program of Jilin Province (20170201005GX) and by the Ministry of Education "Chunhui Plan" (Z2016020). 


\section{REFERENCES}

[1] Guo, Hongqiang, H. He, and X. Xiao. "A Predictive Distribution Model for Cooperative Braking System of an Electric Vehicle." Mathematical Problems in Engineering,2014,(2014-2-9) 2014.3(2014):1-11.

[2] Zhang, Junzhi, et al. "Cooperative control of regenerative braking and hydraulic braking of an electrified passenger car." Proceedings of the Institution of Mechanical Engineers Part D Journal of Automobile Engineering 226.10(2012):1289-1302

[3] Ko, J. W., et al. "Co-operative control for regenerative braking and friction braking to increase energy recovery without wheel lock." International Journal of Automotive Technology., vol. 15, no. 2, pp. $253-$ $262,2014$.

[4] Kwon, M. H., et al. "Cooperative control for friction and regenerative braking systems considering dynamic characteristic and temperature condition," International Journal of Automotive Technology., vol. 17, no. 3, pp. 437-446, 2016

[5] Mutoh, Nobuyoshi. "Driving and Braking Torque Distribution Methods for Front- and Rear-Wheel-Independent Drive-Type Electric Vehicles on Roads With Low Friction Coefficient," IEEE Transactions on Industrial Electronics., vol. 59, no. 10, pp.3919-3933, 2012.

[6] Li, Wenfei, H. Du, and W. Li. "A New Torque Distribution Strategy for Blended Anti-Lock Braking Systems of Electric Vehicles Based on Road Conditions and Driver's Intentions," SAE International., vol. 9 no. 1, pp.107-115, 2016.

[7] Wang, B., Huang, X., Wang, J., Guo, X., and Zhu, X. (2015). A robust wheel slip ratio control design combining hydraulic and regenerative braking systems for in-wheel-motors-driven electric vehicles. Ournal of the Franklin Institute. 352, 2, 577-602

[8] Ren, B., Chen, H., Zhao, H., and Yuan, L. (2016). MPC-based yaw stability control in in-wheel-motored EV via active front steering and motor torque distribution. Mechatronics. 38, 103-114.

[9] Yuan, L., Zhao, H., Chen, H., and Ren, B. (2016). Nonlinear MPC-based slip control for electric vehicles with vehicle safety constraints. Mechatronics. 38, 1-15. 